\title{
Double-bar application decreases postoperative pain after the Nuss procedure
}

\author{
Tomohisa Nagaso, MD, ${ }^{\mathrm{a}}$ Junpei Miyamoto, MD, ${ }^{\mathrm{a}}$ Kiyokazu Kokaji, MD, ${ }^{\mathrm{b}}$ Ryohei Yozu, MD, \\ Hua Jiang, MD, ${ }^{\mathrm{c}}$ HongMei Jin, ${ }^{\mathrm{d}}$ and Tamotsu Tamaki, $\mathrm{PhD}^{\mathrm{e}}$
}

Objective: This biomechanical study aims to elucidate whether additional bar application increases postoperative pain after the Nuss procedure for pectus excavatum.

\begin{abstract}
Methods: Clinical evaluation: The intensity of postoperative pain was compared between patients for whom a single-bar was used (single-bar group: $\mathrm{n}=14$ ) and those for whom double bars (double-bar group: $\mathrm{n}=10$ ) were used to correct the thoracic deformity. The evaluation was performed by referring to the frequency with which local anesthetics were self-injected in a patient-controlled anesthetic system and how many days were needed for the patients to resume ambulation. Theoretical evaluation: An original simulation system for the Nuss procedure was developed by producing 3-dimensional finite element analysis models from computed tomographic data of patients with pectus excavatum. With this system, single-bar and double-bar placement was simulated separately for the thorax models of the double-bar group. The stresses occurring on the thoraces were then compared between the two situations.
\end{abstract}

Results: Clinical evaluation: Self-injection of local anesthetic was more frequent for the single-bar group than for the double-bar group; single-bar patients restarted ambulation later than the double-bar group. Theoretical evaluation: Stresses on the thoraces were smaller when double bars were applied than when a single bar was applied.

Conclusions: Performing double-bar placement decreases postoperative pain. Therefore, surgeons should not hesitate to perform double-bar correction in patients in whom the deformity extends to multiple intercostal spaces, requiring correction of the thorax shape at multiple sites. (J Thorac Cardiovasc Surg 2010;140:39-44)

Supplemental material is available online.

In the Nuss procedure for pectus excavatum cases in which thorax deformities extend to multicostal levels, single-bar insertion at one intercostal space does not achieve optimal outcomes. Single-bar insertion may correct the deformity of the regions close to the bar, but the correction effect often does not reach distant regions, leaving the deformity of these regions uncorrected. For these cases, additional bars should be

\footnotetext{
From the Departments of Plastic and Reconstructive Surgery ${ }^{\mathrm{a}}$ and Cardiovascular Sur-

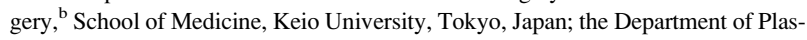
tic Surgery, ${ }^{c}$ Shanghai Second Military Medical College, ChangZheng Hospital, Shanghai, China; and the TOYOTA Central Research Institute, ${ }^{\mathrm{d}}$ Nippon Institute of Technology, ${ }^{\mathrm{e}}$ Nippon, Japan.

Part of the present study was supported by a Grant-in-Aid for Scientific Research from the Ministry of Education, Culture, Sports, Science and Technology of Japan (C21500453).

Disclosures: None.

Received for publication June 19, 2009; revisions received Nov 6, 2009; accepted for publication Dec 13, 2009; available ahead of print April 5, 2010.

Address for reprints: Tomohisa Nagasao, MD, Department of Plastic and Reconstructive Surgery, Keio University Hospital, Shinjuku-Ward Shinanomachi 35, Tokyo, Japan (E-mail: nagasao@sc.itc.keio.ac.jp).

$0022-5223 / \$ 36.00$

Copyright $(c) 2010$ by The American Association for Thoracic Surgery

doi:10.1016/j.jtcvs.2009.12.027
}

used. ${ }^{1,2}$ Application of additional bars exerts dynamic forces to the thorax at multiple sites, which is advantageous for the correction of thoracic deformity extending to wide regions.

On the other hand, potential disadvantages of additional bars should also be considered before using additional bars in actual clinical cases. For instance, increasing the number of correction bars might aggravate patients' postoperative pain. Considerable stress works on the costal cartilages and the rib supporting the correction bar. $^{3}$ Therefore, it would be natural to assume that involving more ribs and cartilages as support for bars increases pain. Alternatively, it is also rational to assume that pain would instead be reduced by application of additional bars, since the force necessary to correct the deformity is distributed to more ribs and costal cartilages.

Postoperative pain—one of the most common complications of the Nuss procedure - causes difficulty in respiration, occasionally leading to pneumonia. ${ }^{4,5}$ Therefore, we perceive that clarifying whether or not application of additional bars increases postoperative pain should provide clinically significant information to surgeons in preparation for the Nuss procedure. The present study aims to elucidate this issue.

In the first part of the present study, postoperative pain is compared between patients in whom a single bar was used and those in whom a double bar procedure was used. In 


\section{Abbreviation and Acronym \\ $\mathrm{CT}=$ computed tomography}

the latter part of the study, differences in stress occurrence patterns are evaluated between single-bar and double-bar applications using finite element analysis on simulated thoraces.

\section{MATERIALS AND METHODS \\ Evaluation of Pain in Clinical Cases}

Study sample. Twenty-four patients who received the Nuss procedure for pectus excavatum at our institutes between April 2003 and May 2009 were involved in the study. All 24 patients received the Nuss procedure as the first major operation in their lives. The purpose and design of the present study were explained preoperatively to all patients, and every part of the present study was conducted under the approval of these patients and the Institutional Review Board of the Department of Plastic Surgery of Keio University Hospital. None of the patients had with a diagnosis of psychologic diseases or mental retardation. For 14 patients (10 men and 4 women), a single bar was placed at the fifth intercostal space to correct deformity of the thorax. These patients were categorized as the single-bar group. For 10 patients ( 7 men and 3 women), two correction bars were placed at the fifth and sixth intercostal spaces. These patients were categorized as the doublebar group. Whether a single bar or double bars were used for each patient was decided during the operation. In all patients, one bar was first placed at the fifth intercostal space. Then a second bar was additionally applied at the sixth intercostal space for patients in whom at least one of the following two situations was present: (1) when the lower part of the thoraces remained uncorrected, an additional bar was applied to improve the lower thorax shape; (2) when considerable resistance was detected during the insertion of the first bar and it was perceived that the thorax was too hard to be supported with one bar, an additional bar was applied to provide sufficient stability to the corrected thoraces. No statistical differences in the patients' ages $(17.8 \pm 8.2$ years for the single-bar group and $19.2 \pm$ 7.4 years for the double-bar group) or Haller indexes ${ }^{6}(6.2 \pm 3.0$ for the single-bar group and $5.8 \pm 2.8$ for the double-bar group) were present between the two groups. The operation time was significantly longer $(P<.0001)$ for the double-bar group (90.2 \pm 14.7 minutes $)$ than for the single-bar group (63.8 \pm 7.9 minutes). None of the 24 patients had serious pneumonia, atelectasis, or infection of the wounds postoperatively.

Evaluation of postoperative pain. The degree of postoperative pain was evaluated referring to the following two items.

INJECTION FREQUENCY OF EPIDURAL ANESTHETIC. For all patients, an epidural anesthetic catheter was placed at the Th5-Th6 level before the operation. Postoperative pain was controlled by patient-controlled anesthesia through these spinal catheters for at least 3 postoperative days. An anesthetic cocktail was prepared by mixing $0.12 \%$ ropivacaine hydrochloric (anapain) and fentanyl citrate (fentanyl) so that the density of fentanyl citrate was $4.2 \mu \mathrm{g} /$ $\mathrm{mL}$. The anesthetic cocktail was continuously injected through the epidural catheter at the rate of $0.42 \mu \mathrm{g} / \mathrm{kg}$ per hour. When patients felt pain despite this continuous injection, they could inject additional anesthetic by pushing buttons at their fingertips. A $0.168 \mu \mathrm{g} / \mathrm{kg}$ anesthetic was administered by each button-push. The minimum interval for the additional injections was set as 20 minutes. Namely, the buttons were arranged not to respond if pushed within 20 minutes of the last additional injection. The postoperative pain for each patient was quantified by counting how many times the patient used the additional injection system in the first 48 hours after the operation. During this period, no other medications - including oral drug administration, usage of suppositories, or systemic injections-were used to control postoperative pain. This quantification parameter was defined as "injection frequency.',

Postoperative DAY OF RESTARTing ambulation. With each patient, the time required to restart ambulation was measured in days. This parameter was defined as "ambulation restart."

Intergroup comparison. The intensity of postoperative pain was compared between the single-bar group and double-bar group referring to the two parameters - injection frequencies and ambulation restart. Thereby, postoperative pain was compared between the two groups.

\section{Biomechanical Evaluation of Thorax Stresses}

Development of Nuss-procedure simulation system. We developed an original system to evaluate the stresses occurring on the thorax after the Nuss procedure. ${ }^{7}$ In the simulation system, the data of the thorax part are extracted from the computed tomographic (CT) data of patients with pectus excavatum by use of graphic software (Rhinoceros 4.0; Applicraft Co, Tokyo, Japan). The data are further edited using structural analysis software (ANSYS10.0; ANSYS Co, Chicago, Ill) to produce a finite element analysis model for each patient's thorax (Figure E1). Each of the 12 ribs, the sternum, and the 12 vertebrae were modeled using 6-, 18-, and 36-beam elements, respectively, in the simulations. The costal cartilages were modeled using different numbers of beam elements according to morphologic complexity. The first to fifth costal cartilages, sixth to tenth costal cartilages, and eleventh to twelfth costal cartilages were modeled with 5-, 5- to 10-, and 3-beam elements, respectively. Young's moduli were calculated on the basis of the CT density of each patient with the equation of Kopperdahl $(\mathrm{E}=-34.7+3230$ QCT, where $\mathrm{E}$ and QCT mean Young's modulus [in mega-pascals] and CT density [in grams per milliliter], respectively) and were allotted to each component of the thorax. ${ }^{8}$ Thus-obtained, Young's moduli were 1580 to $1920 \mathrm{~kg} / \mathrm{mm}^{2}$ (mean 1750 $\mathrm{kg} / \mathrm{mm}^{2}$ ) for cortical bone, 160 to $200 \mathrm{~kg} / \mathrm{mm}^{2}$ (mean $180 \mathrm{~kg} / \mathrm{mm}^{2}$ ) for cancellous bone, and 60 to $108 \mathrm{~kg} / \mathrm{mm}^{2}$ (mean $88 \mathrm{~kg} / \mathrm{mm}^{2}$ ) for costal cartilage.

The Nuss procedure can be simulated using these models in the following manner (Figure 1). First, three points are marked at the intercostal space where the correction bar is assumed to be placed. These three marking points are as follows: $P$, rib-cartilage junction on the right side; $Q$, the inferior aspect of the sternum; and $R$, rib-cartilage junction on the left side. Elevating $Q$ until it reaches the line connecting $P$ and $R$ simulates application of a correction bar. Under these dynamic conditions, stresses on the thoraces are calculated using the finite element method (Figure E2).

Study sample. For each of the 10 patients included in "Evaluation of Pain in Clinical Cases," simulation models were produced using the abovestated system. Ten models produced in this manner were used for the study. Stress calculation. For each of the 10 thorax simulation models, simulation of the Nuss procedure was conducted under two conditions. In the first condition, single-bar placement at the fifth intercostal space was assumed; in the second condition, placement of two bars at the fifth and sixth intercostal spaces was assumed. The first and second conditions were termed "single-bar application" and "double-bar application," respectively. (It should be noted that single-bar group in "Evaluation of Pain in Clinical Cases" and the single-bar application are different. The single-bar application is a simulated condition for cases in which double bars were placed in actual operation). Stresses occurring on the thoraces were calculated using the simulation system for both single-bar and double-bar applications.

Comparison of stresses. With each of the 10 simulation models, stresses occurring on the thoraces were compared between the singlebar and double-bar applications. The comparison was performed on a ribby-rib basis.

\section{Statistical Methodology}

For all statistical calculation, SPSS version 17.0 (SPSS, Inc, Chicago, Ill) was used. 



FIGURE 1. Simulation of the correction bar insertion. The internal aspect of the sternum $(Q)$ was elevated to reach the line connecting bilateral junctions of the costal cartilages and the ribs $(P$ and $R)$. The figures in the left and right columns indicate the thorax shapes before and after the operation, respectively.

For comparison of the operation time in the subsection "Evaluation of Pain in Clinical Cases," the Student $t$ test was used, because operation times presented a normal distribution. For the comparison of injection frequencies and ambulation restart in the same subsection, a nonparametric test (MannWhitney test) was used inasmuch as these parameters showed skewed distribution.

In the subsection "Biomechanical Evaluation of Thorax Stresses," single-bar application and double-bar application were simulated for the same 10 models. Therefore, a paired test needed to be chosen for the statistical analysis. Furthermore, the stresses showed skewed distributions, requiring a nonparametric test. For these reasons, the paired Wilcoxon test was used for the data comparison.

\section{RESULTS}

\section{Evaluation of Pain in Clinical Cases}

Injection frequency was significantly greater for the single-bar group than for the double-bar group $(P=.013)$. Ambulation restart was also greater for the single-bar group than for the double-bar group $(P=.036)$. These findings indicate that postoperative pain was greater for the former than the latter (Table 1).

\section{Biomechanical Evaluation of Thorax Stresses}

Specific values and statistical differences are demonstrated in Table 2 (The box-plot version of these data is

TABLE 1. Comparison of postoperative pain parameters

\begin{tabular}{ccccccc}
\hline & Single-bar group & & \multicolumn{2}{c}{ Double-bar group } & \\
\cline { 2 - 3 } & Mean & Range & & Mean & Range & $\begin{array}{c}\text { Statistical } \\
\text { significance }\end{array}$ \\
\hline $\begin{array}{c}\text { Injection } \\
\text { frequency } \\
\text { (times/2 d) }\end{array}$ & 6.4 & $3-12$ & & 4.2 & $0-8$ & $P=.013$ \\
$\begin{array}{c}\text { Ambulation } \\
\text { restart (d) }\end{array}$ & 4.0 & $1-7$ & & 2.9 & $1-5$ & $P=.036$ \\
\hline
\end{tabular}

demonstrated in Figure E3). Statistically significant differences were present for the fourth and fifth ribs, where the stresses were greater for the single-bar application than for double-bar application. No statistical differences were observed with the stresses occurring on other ribs.

Representative stress-occurrence patterns on a simulated thorax are graphically demonstrated in Figure 2. Stresses tend to be concentrated with the single-bar application, whereas stresses are distributed with the double-bar application.

\section{DISCUSSION}

Although the Nuss procedure is an effective technique for the correction of pectus excavatum in that this procedure enables correction of thoracic deformities with only minor

TABLE 2. Stresses occurring on each rib

\begin{tabular}{lcccccc}
\hline & \multicolumn{2}{c}{ Single-bar } & & \multicolumn{2}{c}{ Double-bar } & \\
\cline { 2 - 3 } Rib & $\begin{array}{c}\text { Mean } \\
\left(\mathbf{k g} / \mathbf{m m}^{2}\right)\end{array}$ & $\begin{array}{c}\text { Range } \\
\left(\mathbf{k g} / \mathbf{m m}^{2}\right)\end{array}$ & & $\begin{array}{c}\text { Mean } \\
\left(\mathbf{k g} / \mathbf{m m}^{\mathbf{2}}\right)\end{array}$ & $\begin{array}{c}\text { Range } \\
\left(\mathbf{k g} / \mathbf{m m}^{\mathbf{2}}\right)\end{array}$ & $\begin{array}{c}\text { Statistical } \\
\text { differences }\end{array}$ \\
\hline I & 0.88 & $0.52-1.22$ & & 0.92 & $0.54-1.20$ & NS \\
II & 0.86 & $0.48-1.48$ & & 1.04 & $0.56-1.50$ & NS \\
III & 1.40 & $1.20-1.70$ & & 1.44 & $1.14-1.60$ & NS \\
IV & 1.56 & $1.25-2.08$ & & 1.32 & $1.08-1.88$ & $P=.005$ \\
V & 2.10 & $1.30-2.72$ & & 1.74 & $0.96-2.18$ & $P=.0001$ \\
VI & 1.86 & $1.44-2.18$ & & 1.80 & $1.58-2.24$ & NS \\
VII & 1.68 & $1.20-2.18$ & & 1.78 & $1.18-2.10$ & NS \\
VIII & 1.50 & $0.84-1.90$ & & 1.48 & $0.92-1.88$ & NS \\
IX & 1.36 & $0.94-1.85$ & & 1.28 & $0.96-1.86$ & NS \\
X & 0.21 & $0.14-0.26$ & & 0.19 & $0.12-0.28$ & NS \\
XI & 0.22 & $0.18-0.30$ & & 0.24 & $0.19-0.29$ & NS \\
XII & 0.22 & $0.17-0.26$ & & 0.23 & $0.16-0.27$ & NS \\
\hline
\end{tabular}

NS, Not significant. 


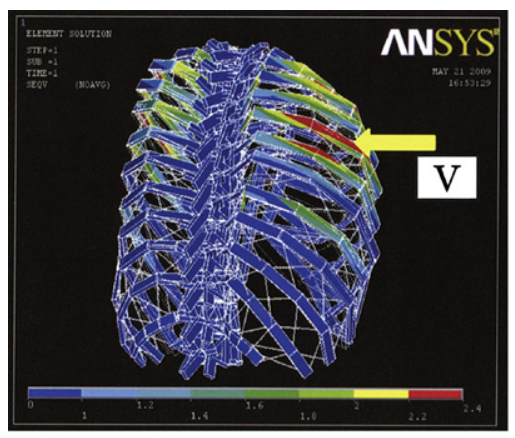

Single-Bar Insertion



Double-Bar Insertion

FIGURE 2. Differing stress-occurrence patterns of single-bar (left) and double-bar applications (right). With single-bar application, stress tends to concentrate on the fifth rib (arrow), whereas stresses are distributed widely across the thorax with double-bar application. $V$, the fifth rib.

incisions and simple techniques, 9,10 the procedure is not free from complications. ${ }^{11-13}$ Postoperative pain is one of its most common complications. ${ }^{14,15}$ In the Nuss procedure, deformed costal cartilages and sternums are forcibly transformed by the correction bar. ${ }^{3}$ Because of this dynamic force, postoperative pain develops in the thoracic region. When the pain is extremely serious, it could even become a causative factor for life-threatening complications-such as pneumonia or respiratory disturbance. Furthermore, when the postoperative pain is intolerable, some patients even require premature removal of the correction bar despite successful correction of the thorax's shape. To avoid these complications, one should take care to minimize patients' postoperative pain. In our effort to achieve this purpose, we were interested in how the details of the proceduresuch factors as the number of correction bars, intercostal levels at which the bars are placed, and the curvature of the correction bars-affect the postoperative pain. We assumed that if we could clarify the relationship between these complicated factors and the degree of postoperative pain, the knowledge would be of some help for surgeons in detailed planning of the operation referring to patient's personality and tolerance for pain. As the first step to approach this theme, the present study focuses on the relationship between the number of correction bars and postoperative pain.

Pain evaluation of clinical cases demonstrates that injection frequency was significantly greater and patients restart ambulation later for the single-bar group than for the double-bar group, indicating that postoperative pain is more serious with the single-bar group than with the double-bar group. In evaluating the meaning of this finding, it should be noted that these two groups were not categorized randomly, although they were matched in terms of male/female proportion, age, and Haller indices. As explained in Materials and Methods, the second bar was applied for patients in whom the deformity of the lower part of the thorax was not fully corrected by the first bar's placement or for patients in whom considerable resistance was detected during the bar's placement. In other words, the thoraces belonging to the double-bar group were harder or presented more severe deformity than those belonging to the single-bar group. This indication might have biased the sample selection. To make the statistical comparison impeccable, one should perform single- or double-bar application randomly.

Nevertheless, we do not believe the potential bias in the sample selection makes the findings meaningless. Compared with flexible or locally deformed thoraces, greater stresses are expected to occur on hard or widely deformed thoraces when correction bars are applied to them. Therefore, hardness or extensive deformity of the thoraces present in the second-bar group should increase, rather than decrease, pain. However, postoperative pain was less serious for the double-bar group than for the single-bar group. If the categorization of the single-bar or double-bar groups had been performed randomly, differences in the seriousness of postoperative pain should have been even more evident.

In the latter half of the study, the validity of the findings in the clinical study was biomechanically evaluated using finite element models. The finite element method is an established study technique in the field of biomechanics and is used for analyses of various medical situations. ${ }^{16-19}$ The analyses of the thorax models demonstrate that the stresses occurring on all ribs are smaller for double-bar application than for singlebar application. This is probably because resistances occurring as a result of elevating the sternum are distributed with the double-bar application.

It is natural that the stress occurring on the fifth rib presents smaller values for the double-bar application than for the single-bar application, since, although the correction bars are supported by only the fifth rib in the single-bar application, they are supported by two ribs in the doublebar application.

In contrast, it is paradoxical that that the stress occurring on the sixth rib does not present statistical difference between the double-bar and single-bar applications. In the double-bar application, resistance forces resulting from the 


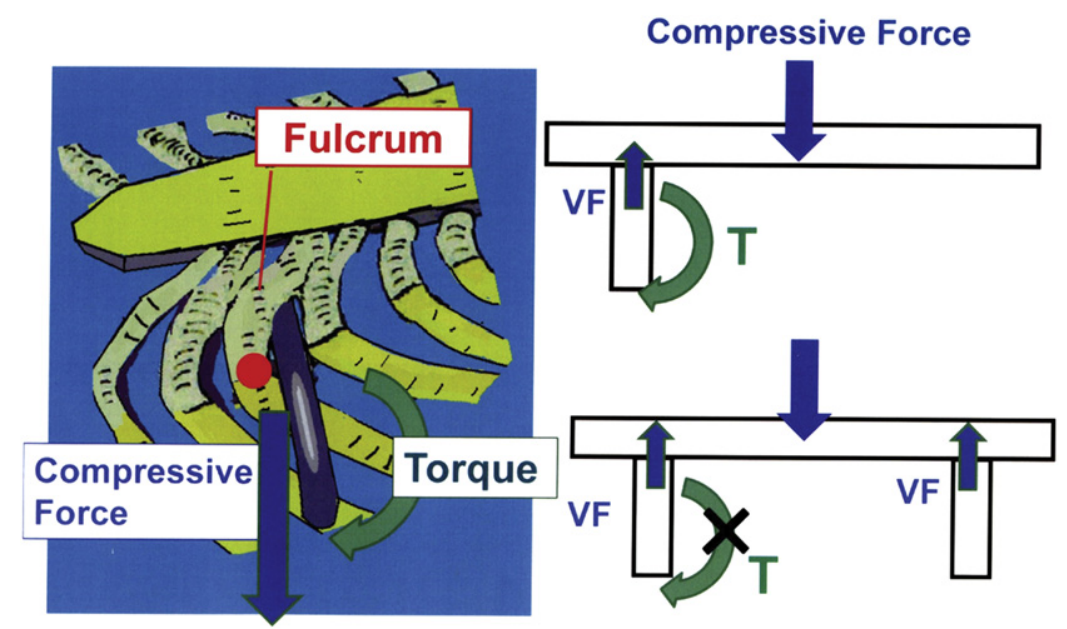

FIGURE 3. Left, Compressive force and torque work on the rib supporting the correction bar. Top right, In the single-strut support, intensified torque develops on the strut. Bottom right, In the double-strut support, torque doe snot develop on the struts.

elevation of the sternum are directly transmitted to the sixth rib through the correction bar, whereas this direct transmission is absent in the single-bar application. For this reason, we had expected the stress on the sixth rib to be greater for the double-bar application than for the single-bar application. However, the biomechanical analysis proved this was not the case.

We present the following hypothesis to explain this paradoxical finding. As demonstrated in Figure 3, left, correction bars are held by the inflection points of the ribs and work to elevate the concave sternum. As the correction bar works, the rib receives mechanical influences in two ways. First, counteracting the sternum's elevation, a compressive force works on the rib in the posterior direction. Second, as the correction bar exerts a cantilever effect with the inflection point as the fulcrum, a torque develops, working to twist the rib.

These mechanical conditions are simulated by two simplified models where a horizontal beam is supported by vertical struts. In the first model, the horizontal beam-which exerts a compressive force-is supported by one vertical strut (Figure 3, top right). In the second model, the horizontal beam is supported by two vertical struts (Figure 3, bottom right). In the first model, the vertical strut needs to resist not only the compressive force $(V F)$, but also the torque developed by the force $(T)$. On the other hand, the torque is offset in the second model, where the vertical struts need only to counteract the compressive force. Because of this torque effect, the stresses working on the vertical struts are much greater for the first model than for the second model.

The first and second models simulate the single-bar and double-bar applications, with the horizontal beam and vertical struts corresponding to the sternum and ribs, respectively. When the sternum is elevated by a single bar, intensified stresses develop on the rib supporting the bar, because of the torque. Therefore, high stresses occur on the fifth rib in the single-bar application. On the other hand, the torque effect is diminished by the double-bar application. This view explains why the sixth ribs do not develop intensified stresses in the double-bar application.

In as much as the degree of postoperative pain is expected to correlate with the stresses occurring on the bone, it would be rational to assume that patients have less pain with double-bar application than with single-bar application.

The findings of the present study indicate that for patients with hard thoraces or widely extending deformity, additional application of the second bar reduces postoperative pain. This finding is clinically important. Application of the second bar is disadvantageous in terms of material costs. Furthermore, as shown in the present study, longer operation time is needed to apply additional bars. This prolonging of operation time may increase infection risks. If the application of the second bar also increases postoperative pain, double-bar application should be avoided. However, the present study proves that double-bar application decreases postoperative pain.

It is therefore concluded that for patients with pectus excavatum in whom the thoraces are too hard to be corrected with only one bar or in whom the thoracic deformity includes multicostal regions, double-bar application is advantageous, not only to correct the thorax shape more effectively, but also to reduce postoperative pain by reducing the stresses on the thoraces. For these patients, surgeons need not be too reluctant to apply the additional bar. When a second bar is necessary for effective correction of thorax shapes, it is recommended that the bar be used without hesitation.

\section{CONCLUSION}

The present study clarifies the influence of double-bar application of the Nuss procedure on postoperative pain. In pain evaluation of clinical cases, it was demonstrated 
that patients who received double-bar placement had less pain than those who had single-bar placement. This finding was supported by the findings of a biomechanical study in which it was demonstrated that the stresses occurring on the thoraces are reduced by double-bar application. Judging from these findings, it is concluded that for patients with pectus excavatum in whom the thoraces are too hard to be corrected with only one bar, or in whom the thoracic deformity includes multicostal regions, double-bar application is advantageous, not only to correct the thorax shape more effectively, but also to reduce postoperative pain by reducing the stresses on the thoraces.

\section{References}

1. Fang FC, Cheng YL, Lee SC, Chen JC, Hsu HH, Tzao C. Clinical experience of Nuss procedure for pectus excavatum in adult female patients. Thorac Cardiovasc Surg. 2008;56:283-6.

2. Park HJ, Lee SY, Lee CS, Youm W, Lee KR. The Nuss procedure for pectus excavatum: evolution of techniques and early results on 322 patients. Ann Thorac Surg. 2004;77:289-95.

3. Weber PG, Huemmer HP, Reingruber B. Forces to be overcome in correction of pectus excavatum. J Thorac Cardiovasc Surg. 2006;132:1369-73.

4. Teh SH, Hanna AM, Pham TH, Lee A, Deschamps C, Stavlo P, et al. Minimally invasive repair for pectus excavatum in adults. Ann Thorac Surg. 2008;85:1914-8.

5. Futagawa K, Suwa I, Okuda T, Kamamoto H, Sugiura J, Kajikawa R, et al. Anesthetic management for the minimally invasive Nuss procedure in 21 patients with pectus excavatum. $J$ Anesth. 2006;20:48-50.

6. Haller JA Jr, Kramer SS, Lietman SA. Use of CT scans in selection of patients for pectus excavatum surgery: a preliminary report. J Pediatr Surg. 1987;22:904-6.

7. Nagasao T, Miyamoto J, Tamaki T, Ichihara K, Jiang H, Taguchi T, et al. Stress distribution on the thorax after the Nuss procedure for pectus excavatum results in different patterns between adult and child patients. J Thorac Cardiovasc Surg. 2007; 134:1502-7.
8. Kopperdahl DL, Pearlman JL, Keaveny TM. Biomechanical consequences of an isolated overload on the human vertebral body. J Orthop Res. 2000;18: 685-90.

9. Nuss D, Kelly REJ, Croitoru DP, Katz ME. A 10-year review of a minimally invasive technique for the correction of pectus excavatum. J Pediatr Surg. 1998;33:545-52.

10. Croitoru DP, Kelly REJ, Goretsky MJ, Gustin T, Keever R, Nuss D. The minimally invasive Nuss technique for recurrent or failed pectus excavatum repair in 50 patients. J Pediatr Surg. 2005;40:181-7.

11. Schalamon J, Pokall S, Windhaber J, Hoellwarth ME. Minimally invasive correction of pectus excavatum in adult patients. J Thorac Cardiovasc Surg. 2006;132: 524-9.

12. Schwabegger AH, Jeschke J, Del Frari B. A rounded dissector to reduce complications in the minimally invasive repair (Nuss) of pectus excavatum in adolescents and adults. Thorac Cardiovasc Surg. 2008;56:118-20.

13. Dzielicki J, Korlacki W, Janicka I, Dzielicka E. Difficulties and limitations in minimally invasive repair of pectus excavatum -6 years experiences with Nuss technique. Eur J Cardiothorac Surg. 2006;30:801-4.

14. Weber T, Mätzl J, Rokitansky A, Klimscha W, Neumann K, Deusch E. Medical Research Society. Superior postoperative pain relief with thoracic epidural analgesia versus intravenous patient-controlled analgesia after minimally invasive pectus excavatum repair. J Thorac Cardiovasc Surg. 2007;134:865-70.

15. Butkovic D, Kralik S, Matolic M, Kralik M, Toljan S, Radesic L. Postoperative analgesia with intravenous fentanyl PCA vs epidural block after thoracoscopic pectus excavatum repair in children. Br J Anaesth. 2007;98:677-81.

16. Yoshioka I, Saiki Y, Ichinose A, Takase K, Takahashi S, Ohashi T, et al. Tagged cine magnetic resonance imaging with a finite element model can predict the severity of retrosternal adhesions prior to redo cardiac surgery. J Thorac Cardiovasc Surg. 2009;137:957-62.

17. Balaras E, Cha KS, Griffith BP, Gammie JS. Treatment of aortic stenosis with aortic valve bypass (apicoaortic conduit) surgery: an assessment using computational modeling. J Thorac Cardiovasc Surg. 2009;137:680-7.

18. Nagasao T, Nakajima T, Kimura A, Kaneko T, Jin H, Tamaki T. The dynamic role of "Buttress" reconstruction after maxillectomy. Plast Reconstr Surg. 2005;115: 1328-40; discussion 1341.

19. Nagasao T, Miyamoto J, Nagasao M, Ogata H, Kaneko T, Tamaki T, et al. The effect of striking angle on the buckling mechanism in blowout fracture. Plast Reconstr Surg. 2006;117:2373-80; discussion 2381. 

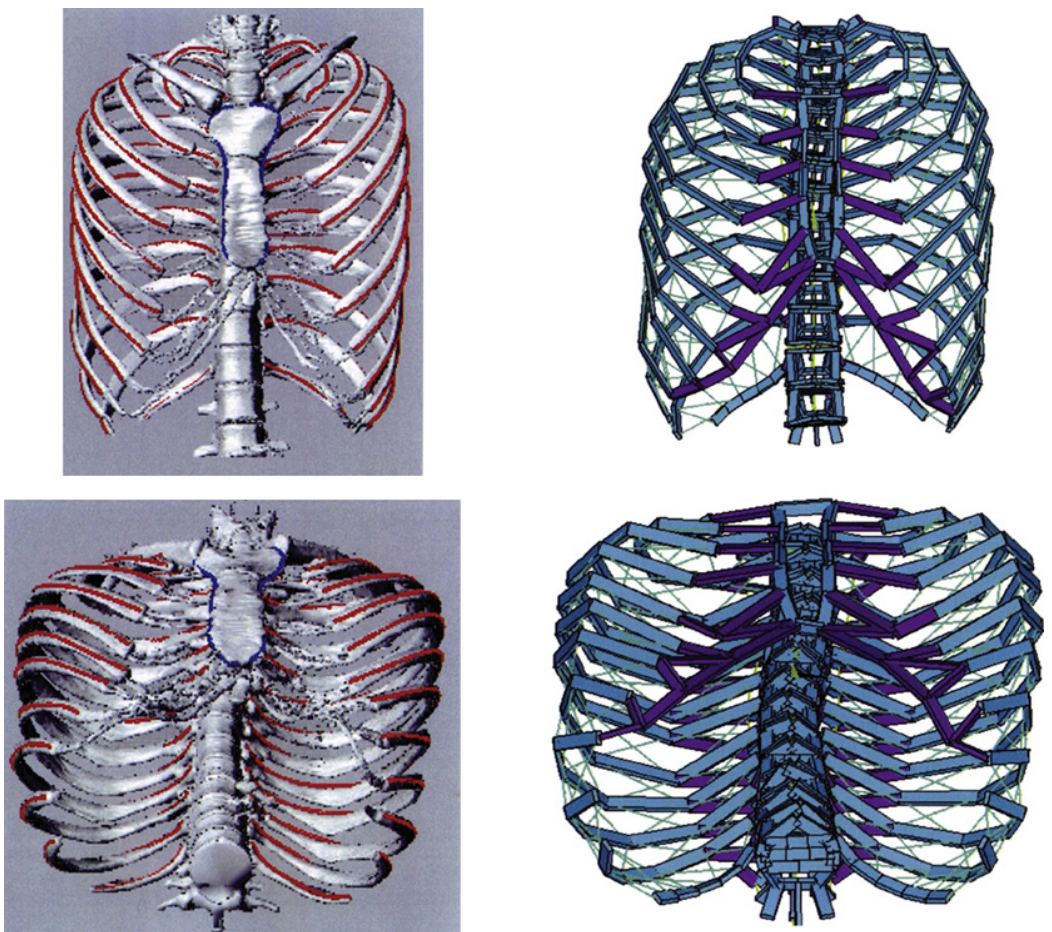

FIGURE E1. On the basis of 3-dimensional computed tomographic data, a finite element model was produced for each patient. Figures in the left and right columns are the original computed tomographic data and the simulated model (viewed from different perspectives), respectively.
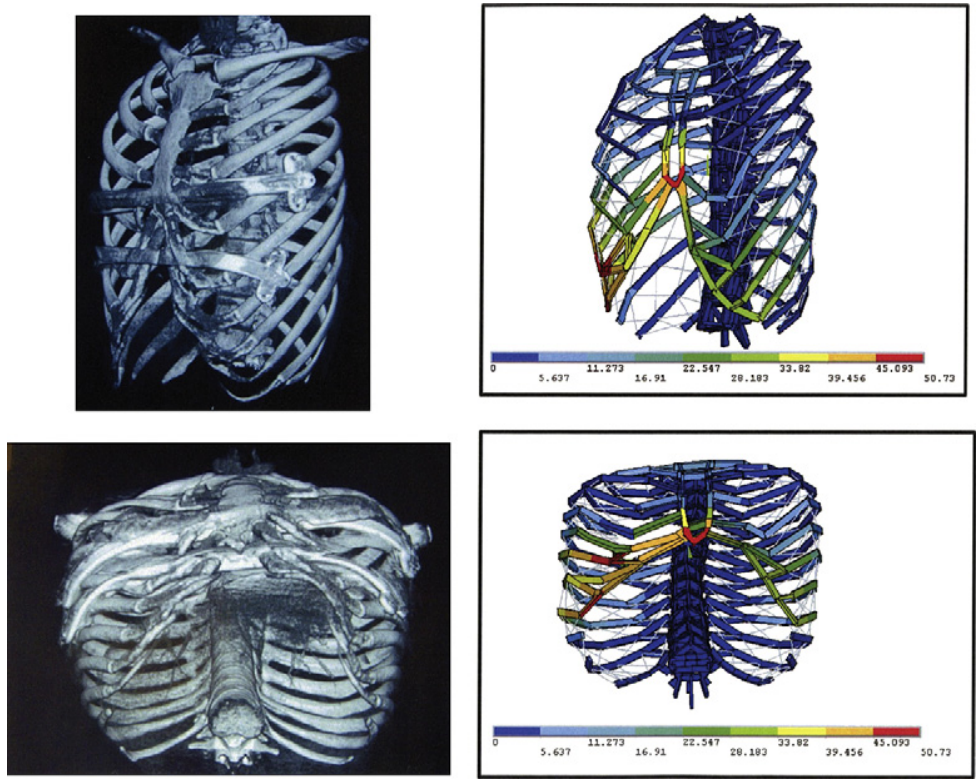

FIGURE E2. A representative result of the simulation: Figures in the left column are computed tomographic data of an actual double-bar application case. Figures in the right column show expected thorax shape, where the degree of correction for each part is demonstrated by color contour mapping. 


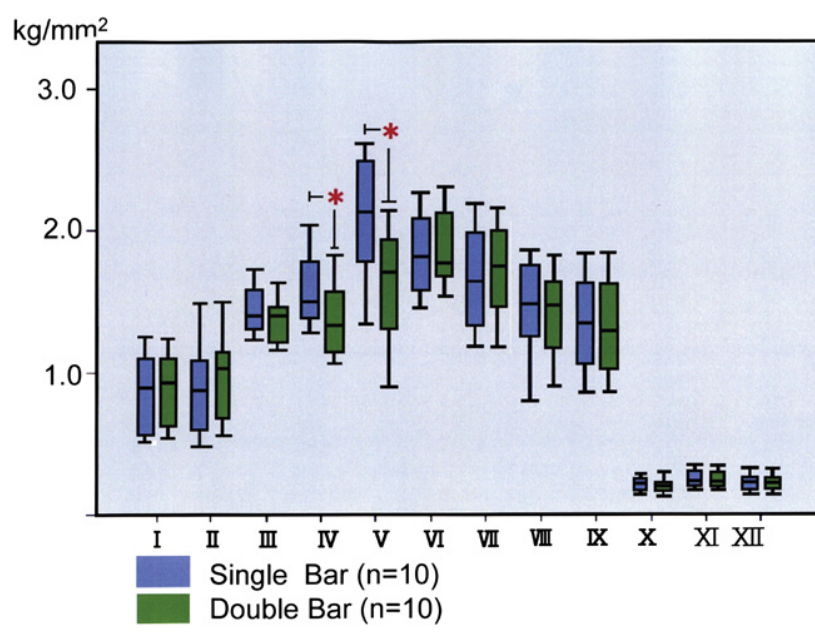

FIGURE E3. Rib -by-rib differences in stress-distribution patterns between single-bar and double-bar applications. The horizontal and vertical axes indicate the rib number and stresses occurring on the corresponding ribs. Asterisks indicate presence of significant differences. The bars inside the boxes indicate the averages of the data. Each box indicates the range in which $59 \%$ of the data are covered. The attaching bars indicate the whole data range. 